\title{
Dioxins in diesel exhaust
}

SIR - Particulates (mainly soot) emitted from diesel engines can be controlled by inserting a ceramic 'regenerative trap' or filter into the exhaust pipe. The filter is cleaned (regenerated) when the carbonaceous deposit ignites and burns away. In real engines, ignition must be promoted either by heating (electrically or by injecting a slug of fuel) or by catalytically burning the deposit. This is achieved by adding an organic metal compound', for example copper, to the fuel. It decreases the quantity of soot formed in the engine and lowers the ignition temperature ${ }^{2}$. We show here, however, that particulate (soot) generation is ameliorated at the expense of increased dioxin emissions.

In the presence of a chlorine donor, dioxins/furans (PCCD/PCCF) can be formed by de novo synthesis in combustion gases as they cool through the range $250-400{ }^{\circ} \mathrm{C}$ (ref. 3). Diesel fuels contain small amounts of chlorine, which is known to form dioxins ${ }^{4}$. Certain metals can act as catalysts in these reformation reactions, copper being the most potent ${ }^{5}$. This raises the possibility that dioxin formation will be increased in a particulate trap system using copper additive in the fuel. To examine this possibility, we performed a series of tests using a small diesel engine driving an electrical generator, operated under nor-
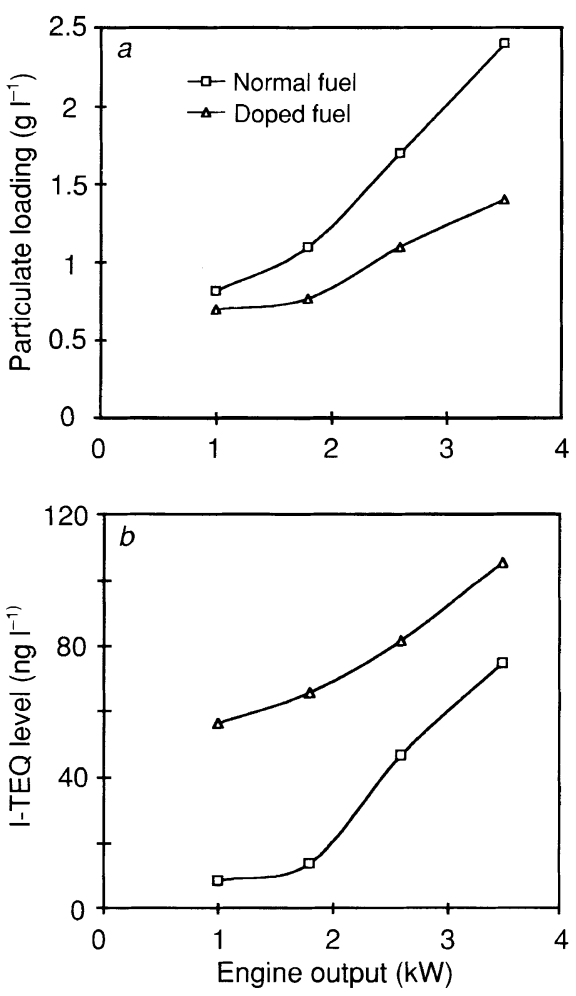

a, Particulate formation at different engine loads; $b$, PCDD/PCDF formation at different engine loads. mal conditions and when fitted with a regenerative soot trap.

We fitted a single-cylinder, 2.0-litre, indirect injection engine, rated at $3.5 \mathrm{~kW}$ nominal output with a cylindrical, commercial-grade cordierite monolith trap (Corning EX 66-100/17). The stock diesel fuel contained 0.9 p.p.m. chlorine as chloride. We prepared doped fuel by mixing $34.4 \mathrm{mg} \mathrm{l}^{-1}$ of copper into the stock fuel. We tested for emissions at various engine loads but constant speed using the stock fuel without the soot trap, and the copper-doped fuel with the soot trap.

The exhaust gases were withdrawn isokinetically through a probe, directed through the trap and into a US EPA method 23 sampling train, modified method 5 (ref. 6). The organic compounds were adsorbed onto specially purified XAD-2 resin which had been previously spiked with a range of stable isotope PCDD and PCDF surrogate standards. We weighed the particulates from the probe, filter, resin cartridge, trap and impinger. The contents from the sample train were extracted with organic solvents, purified and analysed by high-resolution gas chromatography and high-resolution electron impact mass spectrometry.

The figure $(a)$ shows the particulate

loadings of the exhaust gases, recorded as grams per litre of fuel used. The loadings with standard fuel increase by a factor of nearly three over the range of engine output. With copper present, the comparable particulate concentrations fall by 25 to $50 \%$, with better results at higher outputs. This is consistent with the recorded behaviour of doped fuel ${ }^{1}$.

The dioxin/furan results are reported on a toxic equivalent basis (I-TEQ) to allow for the different toxicities of the congeners (see $b$ in the figure). Using the doped fuel, there is a significant increase in dioxin emissions, especially at low load. There are therefore conflicting environmental effects from the addition of copper to diesel fuels.

\section{Clunies-Ross}

\section{B. R. Stanmore}

\section{Department of Chemical Engineering,}

\section{G. J. Millar}

Department of Chemistry,

University of Queensland,

Queensland 4072, Australia

1. Saile, J. A., Monin, G. J. \& Daly, D. T. Worldwide Emission Standards and How to Meet Them 171-181 (Inst. Mech. Eng., London, 1993)

. Bonnefoy, F., Gilot, P. \& Stanmore, B. R. Carbon 32 1333-1339 (1994)

3. Oehme, M., Larssen, S. \& Breik, E. M. Chemosphere 23, 1699-1708 (1991).

4. Bozzelli, J. W., Wu, Y. G. \& Ritter, E. R. Chemosphere 23, 1221-1232 (1991)

5. Gullett, B. K., Bruce, K. R., Beach, L. O. \& Drago, A. M. Chemosphere 25, 1387-1392 (1992).

6. EPA Federal Code of Regulations, Protection of the Environment Title 40, Pts 53-80 (US EPA, 1982).

\section{Evolution of cetacean osmoregulation}

SIR - Cetaceans (whales, dolphins and porpoises) underwent dramatic changes during their evolutionary transformation from four-footed land animals to obligate swimmers. The morphological aspects of this transition have only recently been documented with fossils ${ }^{1,2}$ and provide a striking example of adaptation to a new environment. However, equally remarkable changes must have occurred in aspects of cetacean biology that are not reflected in the gross morphology of fossils. Here, we use oxygen isotope evidence to document the early presence in cetaceans of one of the most extraordinary physiological adaptations to life in the sea: their ability to survive without fresh water. This adaptation made it possible for cetaceans to live offshore early in their evolution and allowed them to disperse across open oceans, sparking a worldwide radiation in the Eocene.

Most mammals cannot survive without a freshwater source, and marine mammals have adopted various osmoregulatory strategies to cope with the salt in their environment. In contrast to some other marine mammals ${ }^{3}$, cetaceans $s^{4,5}$ do ingest sea water, but it is unclear when the cetacean osmoregulatory system adapted to the excess salt load associated with ingesting sea water.

To address this problem, we determined the oxygen isotope composition of phosphate in teeth of early cetaceans. Biogenic phosphate, particularly tooth enamel, appears to be highly retentive of its original oxygen isotope ratio under moderate diagenetic conditions ${ }^{6}$. Mammalian teeth are mineralized in isotopic equilibrium with body water, the isotope composition of which is dominated by ingested water ${ }^{7}$. Thus, the oxygen isotope composition of bone and teeth predominantly reflects that of ingested water. As fresh waters are isotopically lighter than sea water, tooth phosphate of mammals ingesting fresh water will have lower $\delta^{18} \mathrm{O}$ values than the teeth of mammals ingesting sea water ${ }^{8}$. Thus, the isotope composition of the teeth is a tracer of the broad categories (marine versus fresh) of water ingested. Factors in addition to the flux of ingested water may also influence oxygen isotope compositions ${ }^{9,10}$. We therefore tested whether ingested water dominates the isotope composition in cetaceans by analysing modern freshwater and marine species with a wide variety of diets and from a broad geographic range (see figure). The magnitude and direction of the 